Repository of the Max Delbrück Center for Molecular Medicine (MDC) Berlin (Germany)

http://edoc. molc-berlin.de/14422/

\title{
Disorder and residual helicity alter p53-Mdm2 binding affinity and signaling in cells.
}

Borcherds, W., Theillet, F.X., Katzer, A., Finzel, A., Mishall, K.M., Powell, A.T., Wu, H., Manieri, W., Dieterich, C., Selenko, P., Loewer, A., Daughdrill, G.W. 


\section{Disorder and residual helicity alter p53-Mdm2 binding affinity and signaling in cells}

Wade Borcherds ${ }^{1,2,7}$, François-Xavier Theillet ${ }^{3,7}$, Andrea Katzer ${ }^{4,7}$, Ana Finzel ${ }^{4}$, Katie M Mishall ${ }^{1,2}$, Anne Powell ${ }^{1,2}$, Hongwei $\mathrm{Wu}^{1,2}$, Wanda Manieri ${ }^{5}$, Christoph Dieterich ${ }^{6}$, Philipp Selenko ${ }^{3} *$, Alexander Loewer ${ }^{4} *$ \& Gary W Daughdrill $^{1,2} *$

${ }^{1}$ Department of Cell Biology, Microbiology and Molecular Biology, University of South Florida, Tampa, Florida, USA. ${ }^{2}$ Center for Drug Discovery and Innovation, University of South Florida, Tampa, Florida, USA. ${ }^{3}$ In-cell NMR Laboratory, Department of NMR-supported Structural Biology, Leibniz Institute of Molecular Pharmacology (FMP Berlin), Berlin, Germany. ${ }^{4}$ Berlin Institute for Medical Systems Biology, Max Delbrueck Center for Molecular Medicine, Berlin, Germany. ${ }^{5}$ Drug Discovery Department, Moffitt Cancer Center, University of South Florida, Tampa, Florida, USA. ${ }^{6}$ Max Planck Institute for Biology of Ageing, Cologne, Germany. ${ }^{7}$ These authors contributed equally to this work.

*e-mail: selenko@fmp-berlin.de, alexander.loewer@mdc-berlin.de or gdaughdrill@usf.edu

Levels of residual structure in disordered interaction domains determine in vitro binding affinities, but whether they exert similar roles in cells is not known. Here, we show that increasing residual p53 helicity results in stronger Mdm2 binding, altered p53 dynamics, impaired target gene expression and failure to induce cell cycle arrest upon DNA damage. These results establish that residual structure is an important determinant of signaling fidelity in cells.

Intrinsically disordered protein domains often mediate protein-protein interactions that result in disorder-to-order transitions via coupled folding and binding reactions ${ }^{1}$. In addition, many disordered interaction domains exhibit defined levels of transient secondary structure resembling their complex-bound states when free in solution ${ }^{2}$. These levels of residual structure affect binding energies, also by reducing the loss of conformational entropy associated with disorderto-order transitions ${ }^{3}$. Accordingly, higher levels of residual structure in disordered interaction domains increase in vitro binding affinities ${ }^{4,5}$. Here we ask to what extent residual structure contributes to protein binding affinities in cells and whether engineered changes to residual structure affect protein function at the cellular level. To answer these questions, we designed p53 mutants with higher residual helicity within their disordered, N-terminal transactivation domains (TADs) and investigated their effects on cellular Mdm2 binding and p53’s ability to induce 
target gene expression and cell cycle arrest.

p53 is activated by many forms of cellular stress, including DNA double-strand breaks (DSBs) and functions as a major tumor suppressor and cell cycle regulator ${ }^{6}$. In the absence of DNA damage, cellular p53 levels are kept low by targeted proteasomal degradation mediated by the E3 ubiquitin ligase Mdm2, which interacts with p53TAD and subsequently ubiquitinates p53’s C-terminal regulatory domain ${ }^{7}$. Upon DNA damage, post-translational modifications of p53TAD and Mdm2, together with Mdm2 degradation, disrupt the p53-Mdm2 complex. This leads to p53 accumulation and the expression of p53 target genes that regulate DNA repair, cell cycle arrest, senescence or apoptosis ${ }^{8,9}$. One of these target genes is Mdm2, whose expression establishes a negative feedback loop that shapes cellular p53 dynamics and thereby controls cell fate decisions ${ }^{10}$.

In its free form, p53TAD exists in equilibrium between disordered and partially helical conformations $^{11-13}$, whereas residues $19-25$ form a stable amphipathic $\alpha$-helix in the Mdm2 complex $^{14}$ (Fig. 1a). To increase the binding affinity between 553 and Mdm2 without altering the binding interface, we designed p53TAD mutants with higher levels of residual helicity by mutating conserved proline residues flanking the Mdm2 binding site (i.e., Pro12, Pro13 or Pro27) to alanines (Supplementary Results, Supplementary Fig. 1a). Using NMR spectroscopy, we determined that wild-type (WT) p53TAD helicity (28\%) increased to 64\% when we replaced Pro27 with alanine (P27A) (Fig. 1b and Supplementary Fig. 1b). Substituting N-terminal p53TAD prolines (P12A P13A) only had a minor effect on helicity (32\%) and replacing both $\mathrm{N}$ - and C-terminal prolines (P12A P13A P27A, referred to henceforth as P3xA) did not increase helicity beyond the level observed for the P27A mutation (66\%). We performed heteronuclear NOE measurements to confirm that these changes were confined to the Mdm2 binding site and did not extend to other regions of p53TAD (Supplementary Fig. 2a).

Next, we used isothermal titration calorimetry (ITC) to determine whether higher levels of residual helicity in free p53TAD strengthened its interaction with Mdm2 (Supplementary Fig. 2b). p53TAD and p53TAD ${ }^{\text {P12A P13A }}$ bound to Mdm2 with similar affinities, whereas $\mathrm{p} 53 T A D^{\mathrm{P27A}}$ and p53TAD ${ }^{3 \mathrm{xA}}$ displayed approximately tenfold reductions in their dissociation constants ${ }^{15-17}$ (Fig. 1c and Supplementary Fig. 3a). NMR titrations with unlabeled Mdm2 and ${ }^{15} \mathrm{~N}$-labeled $\mathrm{p} 53 \mathrm{TAD}$ and $\mathrm{p53TAD}{ }^{\mathrm{P} 27 \mathrm{~A}}$ produced similar chemical shift changes 
(Supplementary Fig. 3b), which confirmed that both domains bound Mdm2 in structurally equivalent conformations. Results from reciprocal NMR experiments with unlabeled p53TAD and $\mathrm{p}_{3} \mathrm{TAD}^{\mathrm{P} 27 \mathrm{~A}}$ and isotope-labeled Mdm2 further supported this conclusion (Supplementary Fig. 3c). Heteronuclear NOE measurements on isotope-labeled p53TAD and p53TAD ${ }^{\text {P27A }}$ bound to unlabeled Mdm2 additionally revealed that both complexes displayed similar dynamic properties (Supplementary Fig. 4a), suggesting that greater Mdm2 binding indeed resulted from higher levels of residual helicity and not an extended binding interface.

To investigate whether higher p53TAD helicity affected Mdm2 binding and p53 signaling in a cellular context, we downregulated endogenous p53 in MCF7 cells and substituted it with full-length $\mathrm{p} 53, \mathrm{p} 53^{\mathrm{P} 27 \mathrm{~A}}$ or $\mathrm{p} 53^{\mathrm{P3XA}}$ fused to the fluorescent protein Venus

(Supplementary Fig. 4b). We selected clones with similar levels of p53 mRNA and protein expression (Supplementary Fig. 4c) and used time-lapse single-cell fluorescence microscopy to measure the dynamics of nuclear p53 accumulation upon induction of DNA DSBs by ionizing radiation (Supplementary Fig. 4d). Cells expressing WT p53 displayed characteristic oscillatory accumulations with first peaks at $2-3 \mathrm{~h}^{10,18}$, whereas cells expressing $\mathrm{p} 53^{\mathrm{P} 27 \mathrm{~A}}$ and $\mathrm{p} 53^{\mathrm{P} 3 \mathrm{xA}}$ showed earlier and shorter pulses (Fig. 2a). By analyzing the properties of these p53 oscillations, we found that the first pulses in $\mathrm{p}^{\mathrm{P}} 3^{\mathrm{P} 27 \mathrm{~A}}$ and $\mathrm{p} 53^{\mathrm{P} 3 \mathrm{XA}}$ cells displayed consistently shorter widths and ramp times, and lower oscillation pitches, whereas their relative amplitudes were unchanged (Supplementary Fig. 5). Using western blotting, we additionally verified that shorter p53 and Mdm2 pulses in $\mathrm{p} 53^{\mathrm{P} 27 \mathrm{~A}}$ and $\mathrm{p} 53^{\mathrm{P} 3 \times \mathrm{A}}$ cells occurred in all clones, independent of differences in p53 mRNA expression levels and relative protein abundance (Supplementary Fig. 6a). Because phosphorylation of multiple p53 sites regulates intracellular protein accumulation in response to DNA damage ${ }^{9}$, we confirmed that $\mathrm{p} 53, \mathrm{p} 53^{\mathrm{P} 27 \mathrm{~A}}$ and $\mathrm{p} 53^{\mathrm{P} 3 \times \mathrm{A}}$ were modified in a similar manner and that the shortened accumulations of $\mathrm{p} 53^{\mathrm{P} 27 \mathrm{~A}}$ and $\mathrm{p} 53^{\mathrm{P} 3 \mathrm{xA}}$ matched their individual phosphorylation profiles (Supplementary Fig. 6b). We further showed that the phosphorylation rates of WT, $\mathrm{p} 53^{\mathrm{P} 27 \mathrm{~A}}$ and $\mathrm{p} 53^{\mathrm{P} 3 \mathrm{AA}}$ Ser15 and Ser37 with recombinant DNA-PK and by endogenous kinases in MCF7 cell lysates were comparable (Supplementary Fig. 7a).

To address whether changes in cellular p53 dynamics were indeed caused by stronger intracellular binding to Mdm2, we treated the clonal MCF7 lines with Nutlin3a, a smallmolecule inhibitor of the p53-Mdm2 interaction (half-maximum inhibitory concentration ( IC $_{50}$ ) 
$90 \mathrm{nM}$ ) that stabilizes p53 in the absence of DNA damage ${ }^{19}$. In line with previous results, addition of Nutlin3a resulted in the accumulation of WT p53 (Fig. 2b and Supplementary Fig. 7b). Having determined that $\mathrm{p} 53^{\mathrm{P} 27 \mathrm{~A}}$ and $\mathrm{p} 53^{\mathrm{P} 3 \mathrm{xA}}$ exhibit stronger Mdm2 binding in vitro, we predicted a reduction in Nutlin3a’s efficiency to inhibit the Mdm2 interaction and induce p53 accumulation. Accordingly, our experiments showed that tenfold higher Nutlin3a concentrations were needed to detect low levels of p53 in p53 ${ }^{\mathrm{P} 27 \mathrm{~A}}$ and p53 ${ }^{\mathrm{P3xA}}$ cells (Fig. $2 \mathbf{b}$ and

Supplementary Figs. 7c, 8a). To validate that WT and mutant cells had, in principle, the ability to produce equal amounts of p53, we treated them with the proteasome inhibitor MG132 (ref. 20), thus preventing Mdm2-mediated degradation altogether. We observed similar accumulations of cellular p53 in all cases (Fig. 2c and Supplementary Fig. 8a).

Next, we asked how altered p53 accumulations affected target gene expression. Mediators of the p53 response pathway include the cell-cycle inhibitor p21 and the proapoptotic Bcl2 family member Bax ${ }^{21,22}$. Because p53 dynamics contribute to the strength and specificity of target gene expression ${ }^{23}$, we expected $\mathrm{p} 53^{\mathrm{P} 27 \mathrm{~A}}$ and $\mathrm{p} 53^{\mathrm{P} 3 \mathrm{xA}}$ cells to display reduced levels of $\mathrm{p} 21$ and Bax in response to DNA damage. We quantified target mRNA by qPCR after $16 \mathrm{~h}$ of radiation-induced DNA damage and found considerably higher amounts in cells containing WT versus mutant p53 (Fig. 3a and Supplementary Fig. 8b). To confirm that reduced p21 expression in these cells was due to changes in cellular p53 dynamics, we induced DNA damage in the presence of low amounts of Nutlin3a, which has been shown to prolong damage-induced accumulation of WT p53 (ref. 23). In p53 ${ }^{\mathrm{P} 27 \mathrm{~A}}$ and p53 ${ }^{\mathrm{P3xA}}$ cells, addition of Nutlin3a increased the durations of p53 pulses to WT levels (Supplementary Fig. 9a), as competitive inhibition of the $\mathrm{p} 53-\mathrm{Mdm} 2$ interaction by the drug synergized with the radiation-induced post-translational modifications of both proteins. Prolonged accumulations of mutant p53 also rescued p21 expression (Supplementary Fig. 9b). We extended our analyses to genome-wide RNA sequencing (RNA-seq) measurements of mRNA transcripts in WT and mutant cells. For 62 validated p53 target genes, we detected lower mRNA levels in p53 ${ }^{\mathrm{P} 27 \mathrm{~A}}$ and $\mathrm{p} 53^{\mathrm{P} 3 \mathrm{xA}}$ cells in the absence of DNA damage (Fig. 3b), which is in agreement with the lower basal amounts of mutant p53 (ref. 24). By analyzing RNA-seq data $16 \mathrm{~h}$ after DNA damage, we confirmed that p53 target genes were efficiently upregulated in WT cells, whereas transcript levels remained lower in p53 ${ }^{\mathrm{P} 27 \mathrm{~A}}$ and p53 ${ }^{\mathrm{P} x \mathrm{~A}}$ cells. Non-p53 target genes were unaffected (Supplementary Fig. 9c). By quantifying the relative differences in mRNA fold increases of target genes we found 
that the individual induction levels were consistently greater in WT-, than in $\mathrm{p}^{\mathrm{P}} 3^{\mathrm{P} 27 \mathrm{~A}}$ and $\mathrm{p} 53^{\mathrm{P} 3 \mathrm{xA}}$ -cells (Supplementary Fig. 9d).

Having determined that $\mathrm{p} 53^{\mathrm{P} 27 \mathrm{~A}}$ and $\mathrm{p} 53^{\mathrm{P} 3 \mathrm{xA}}$ compromised $\mathrm{p} 53$-dependent target gene expression, we asked whether these perturbations also altered cell fate. We performed flow cytometry experiments on WT and mutant cells after $16 \mathrm{~h}$ of radiation-induced DNA damage and determined cell cycle distributions. At this time point, cells either arrest in G1 primarily through p53-dependent expression of p21 or in G2 through p53-independent mechanisms ${ }^{25}$. Because p53 ${ }^{\mathrm{P} 27 \mathrm{~A}}$ and $\mathrm{p} 53^{\mathrm{P} 3 \mathrm{xA}}$ cells had reduced levels of p21 expression, we expected fewer cells to arrest in G1. Indeed, we measured a 50\% reduction in G1 arrest for $\mathrm{p}^{5} 3^{\mathrm{P} 27 \mathrm{~A}}$ and $\mathrm{p} 53^{\mathrm{P} 3 \mathrm{xA}}$ cells compared to WT p53, which was similarly displayed in all clonal lines (Fig. 3c and Supplementary Fig. 9e).

In summary, we show that residual helicity of p53TAD determines the binding affinity to Mdm2 in vitro and in cells. Conserved proline residues outside the Mdm2 binding site preserve these defined levels of helicity, which is ultimately required for productive p53 signaling. Alanine substitutions that induce higher p53TAD helicity lead to stronger Mdm2 binding, shorter p53 pulses and more rapid degradation in response to DNA damage, which impairs p53’s capacity to induce target gene expression and, eventually, its ability to promote cell cycle arrest. These results imply that disorder and residual helicity of p53TAD have an important role in determining the balance between binding affinities and binding specificities within the p53signaling network.

\section{METHODS}

Methods and any associated references are available in the online version of the paper.

\section{References}

1. Wright, P.E. \& Dyson, H.J. Linking folding and binding. Curr. Opin. Struct. Biol. 19, 31-38 (2009).

2. Marsh, J.A., Teichmann, S.A. \& Forman-Kay, J.D. Probing the diverse landscape of protein flexibility and binding. Curr. Opin. Struct. Biol. 22, 643-650 (2012). 
3. Frederick, K.K., Marlow, M.S., Valentine, K.G. \& Wand, A.J. Conformational entropy in molecular recognition by proteins. Nature 448, 325-329 (2007).

4. Drobnak, I. et al. Energetic basis of uncoupling folding from binding for an intrinsically disordered protein. J. Am. Chem. Soc. 135, 1288-1294 (2013).

5. Iešmantavičius, V., Dogan, J., Jemth, P., Teilum, K. \& Kjaergaard, M. Helical propensity in an intrinsically disordered protein accelerates ligand binding. Angew. Chem. Int. Edn Engl. 53, 1548-1551 (2014).

6. Vousden, K.H. \& Lane, D.P. p53 in health and disease. Nat. Rev. Mol. Cell Biol. 8, 275-283 (2007).

7. Wu, X., Bayle, J.H., Olson, D. \& Levine, A.J. The p53-mdm-2 autoregulatory feedback loop. Genes Dev. 7, 1126-1132 (1993).

8. $\quad$ Kruse, J.P. \& Gu, W. Modes of p53 regulation. Cell 137, 609-622 (2009).

9. Vousden, K.H. \& Prives, C. Blinded by the light: the growing complexity of p53. Cell 137, 413-431 (2009).

10. Lahav, G. et al. Dynamics of the p53-Mdm2 feedback loop in individual cells. Nat. Genet. 36, 147-150 (2004).

11. Lee, H. et al. Local structural elements in the mostly unstructured transcriptional activation domain of human p53. J. Biol. Chem. 275, 29426-29432 (2000).

12. Vise, P.D., Baral, B., Latos, A.J. \& Daughdrill, G.W. NMR chemical shift and relaxation measurements provide evidence for the coupled folding and binding of the p53 transactivation domain. Nucleic Acids Res. 33, 2061-2077 (2005).

13. Wells, M. et al. Structure of tumor suppressor p53 and its intrinsically disordered Nterminal transactivation domain. Proc. Natl. Acad. Sci. USA 105, 5762-5767 (2008).

14. Kussie, P.H. et al. Structure of the MDM2 oncoprotein bound to the p53 tumor suppressor transactivation domain. Science 274, 948-953 (1996).

15. Li, C. et al. Systematic mutational analysis of peptide inhibition of the p53MDM2/MDMX interactions. J. Mol. Biol. 398, 200-213 (2010). 
16. Mittal, J., Yoo, T.H., Georgiou, G. \& Truskett, T.M. Structural ensemble of an intrinsically disordered polypeptide. J. Phys. Chem. B 117, 118-124 (2013).

17. Zondlo, S.C., Lee, A.E. \& Zondlo, N.J. Determinants of specificity of MDM2 for the activation domains of p53 and p65: proline27 disrupts the MDM2-binding motif of p53. Biochemistry 45, 11945-11957 (2006).

18. Batchelor, E., Mock, C.S., Bhan, I., Loewer, A. \& Lahav, G. Recurrent initiation: a mechanism for triggering p53 pulses in response to DNA damage. Mol. Cell 30, 277289 (2008).

19. Vassilev, L.T. et al. In vivo activation of the p53 pathway by small-molecule antagonists of MDM2. Science 303, 844-848 (2004).

20. Maki, C.G., Huibregtse, J.M. \& Howley, P.M. In vivo ubiquitination and proteasomemediated degradation of p53(1). Cancer Res. 56, 2649-2654 (1996).

21. el-Deiry, W.S. et al. WAF1, a potential mediator of p53 tumor suppression. Cell 75, 817-825 (1993).

22. Miyashita, T. \& Reed, J.C. Tumor suppressor p53 is a direct transcriptional activator of the human bax gene. Cell 80, 293-299 (1995).

23. Purvis, J.E. et al. p53 dynamics control cell fate. Science 336, 1440-1444 (2012).

24. Sablina, A.A. et al. The antioxidant function of the p53 tumor suppressor. Nat. Med. 11, 1306-1313 (2005).

25. Toettcher, J.E. et al. Distinct mechanisms act in concert to mediate cell cycle arrest. Proc. Natl. Acad. Sci. USA 106, 785-790 (2009).

\section{Acknowledgments}

We thank P. Schmieder and M. Beerbaum for excellent NMR infrastructure maintenance (FMP Berlin) and W. Chen for Illumina sequencing (Max Delbrueck Center). This research was supported by the Deutsche Forschungsgemeinschaft (Emmy Noether grant PS1794/1-1 to P.S.), the Association pour la Recherche contre le Cancer (postdoctoral fellowship to F.X.T.), the European Union FP7 (Marie Curie CIG to A.L.), the American Cancer Society (RSG-07-289-01-GMC to G.W.D.) and the National Science Foundation (MCB-0939014 to G.W.D.). Protein NMR resources at the University of South Florida were provided by the Center for Drug Discovery and Innovation. 


\section{Author contributions}

W.B., F.-X.T. and H.W. collected and analyzed NMR data. W.B., K.M.M. and A.P. prepared p53TAD and Mdm2 protein samples, F.-X.T., K.M.M. and A.P. collected and analyzed ITC data. W.M. provided the protocol for Mdm2 expression and purification. F.-X.T. and A.K. generated and analyzed mutant p53 cell lines, A.F. performed RNAseq experiments, and C.D. provided bioinformatics analysis. F.-X.T. and W.B. assisted in the writing of the manuscript. P.S., A.L. and G.W.D. conceived the study, supervised the research, analyzed the data, prepared the figures and wrote the manuscript.

\section{Competing financial interests}

The authors declare no competing financial interests.

\section{Additional information}

Supplementary information is available in the online version of the paper. Reprints and permissions information is available online at http://www.nature.com/reprints/index.html. Correspondence and requests for materials should be addressed to P.S., A.L. or G.W.D. 


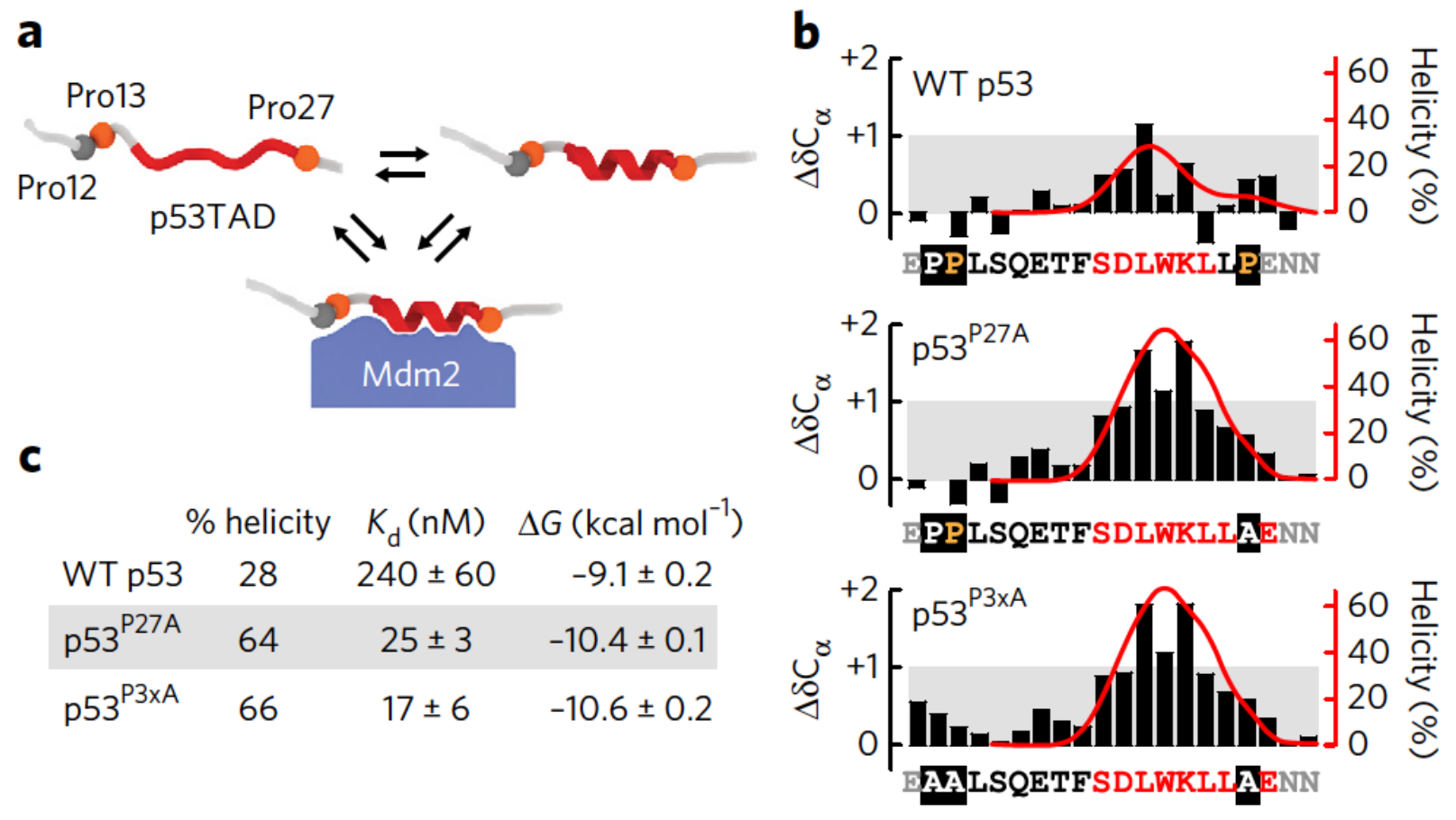

Figure 1 | Transient p53TAD helicity determines the binding affinity to Mdm2. (a)

Schematic overview of the p53TAD-Mdm2 interaction showing equilibria between disordered, helical and bound forms of p53TAD. (b) Positive C $\alpha$ random-coil chemical shift deviations $\left(\delta \Delta \mathrm{C}_{\alpha}\right)$ denote levels of helicity within WT and mutant p53TAD. Red curves show percentage helicity estimates as determined with the d2D method. Conserved prolines (orange) flanking the Mdm2 binding site are highlighted. (c) Mdm2 binding affinities of WT and mutant p53TADs as measured by ITC. 

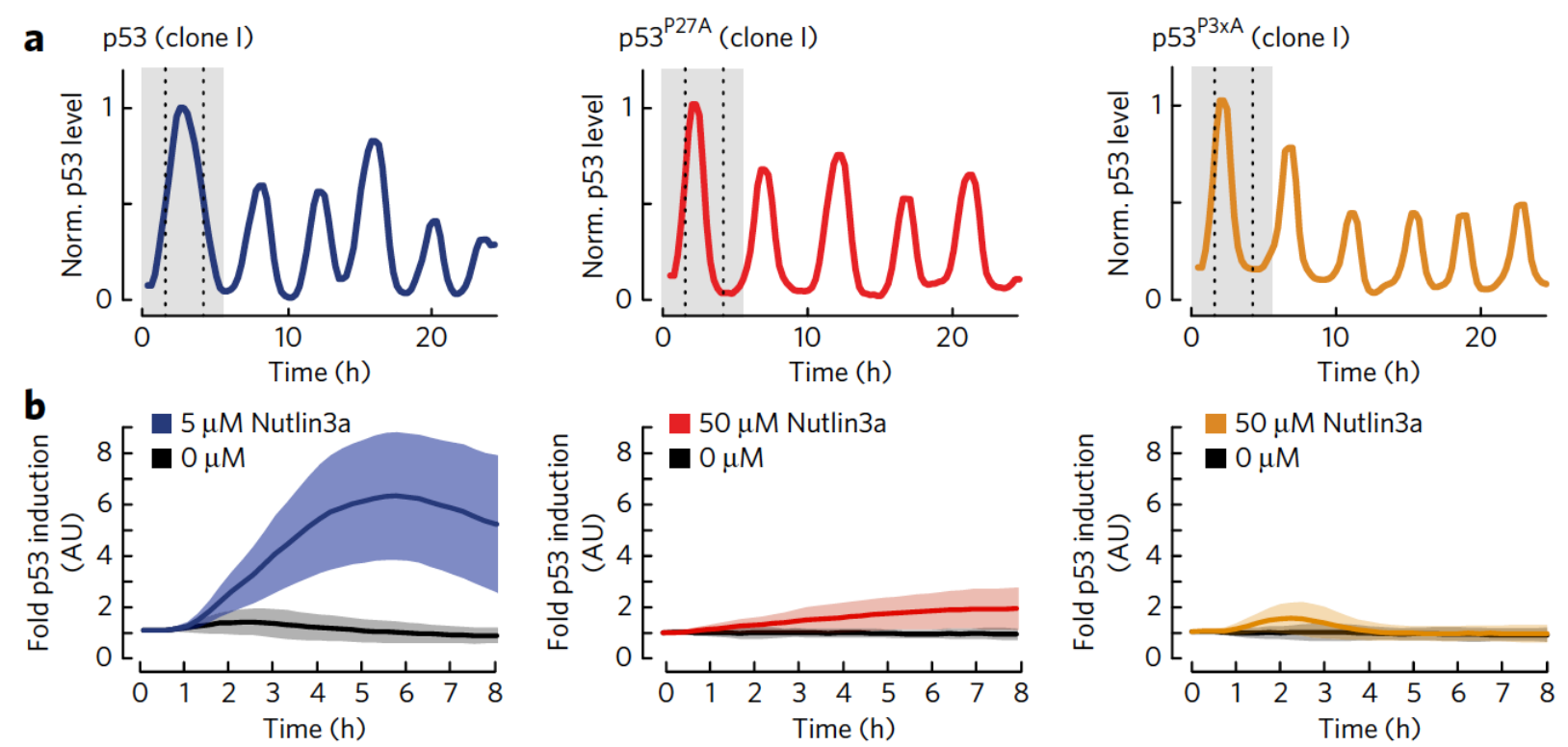

Figure 2 | Altered cellular dynamics of helical p53TAD mutants. (a) Exemplary traces of oscillating p53 protein levels in response to $\gamma$-radiation ( $\gamma$-IR)-induced DNA DSBs. Shaded areas and dashed lines, respectively, indicate pulse duration and width at half maximal peak height in WT p53 cells as reference. For live-cell imaging, at least two independent cell cultures were irradiated analyzed. (b) Protein levels of WT and mutant p53 upon Nutlin3a treatment measured by live-cell fluorescence imaging. Solid lines indicate the mean, and shaded areas represent the s.d. of accumulated responses. $n>60$ cells per condition. a.u., arbitrary units. (c) Protein levels of WT and mutant p53 upon proteasome inhibition with MG132. Solid lines indicate the mean, and shaded areas represent the s.d. of accumulated responses. $n>60$ cells per condition. 

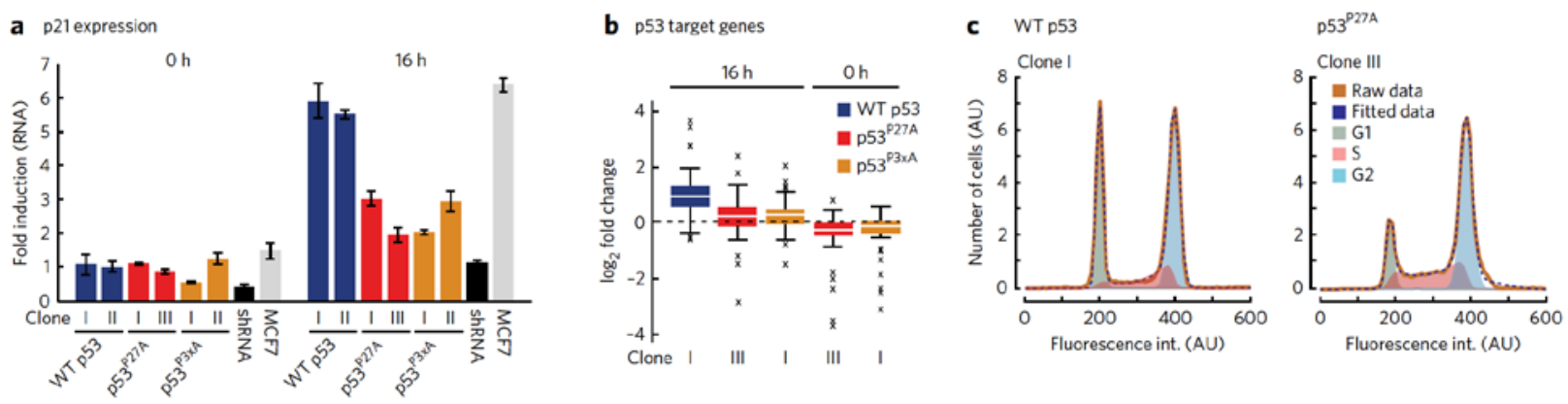

Figure 3 | Altered target gene expression- and cell-cycle profiles of helical p53TAD

mutants. (a) Measured differences in p21 mRNA levels in cells carrying WT and mutant p53 before and $16 \mathrm{~h}$ after DSB induction. p21 levels with background p53 after shRNA knockdown (black) and with endogenous p53 in non-manipulated MCF7 cells (gray). Actin serves as the internal standard for mRNA quantifications. Error bars represent s.d. of technical replicates. Data is representative for duplicate experiments. a.u., arbitrary units. (b) Distributions of log2-fold changes of sixty-two differentially expressed p53 target genes before and after DSB induction. Data are normalized to expression levels of each gene in p53 WT cells before irradiation $(0 \mathrm{~h})$. White lines indicate the median of distribution, boxes include data between the $25^{\text {th }}$ and $75^{\text {th }}$ percentile. Whiskers extend to maximum values that are within 1.5 times the interquartile range; crosses represent outliers. Samples were sequenced on two lanes (rapid run mode); sequencing depth was 250 million reads. (c) Distributions of DNA contents and fitted cell cycle stages as measured by flow cytometry in WT p53 and p53 ${ }^{\mathrm{P} 27 \mathrm{~A}}$ cells, $16 \mathrm{~h}$ after DSB induction. Data is representative for triplicate experiments. 


\section{Online Methods}

\section{Protein purification.}

Uniformly ${ }^{15} \mathrm{~N}$-labeled or ${ }^{15} \mathrm{~N}$ - and ${ }^{13} \mathrm{C}$-labeled and unlabeled samples of WT and mutant versions of human p53TAD (residues 1-73) were prepared as previously described ${ }^{12}$. Samples of human Mdm2, corresponding to residues 17-125, were expressed using pGEX-6p-2 vectors in BL21(DE3) cells grown in M9 medium. Induction with $1 \mathrm{mM}$ IPTG was carried out at $\mathrm{OD}_{600}$ of 0.8 for $5 \mathrm{~h}$ at $25^{\circ} \mathrm{C}$. Cultures were centrifuged at $11,000 \mathrm{~g}$ and frozen at $-80^{\circ} \mathrm{C}$. Pellets were resuspended in GST binding buffer ( $50 \mathrm{mM}$ Tris-HCl, $300 \mathrm{mM} \mathrm{NaCl}, 2.5 \mathrm{mM}$ EDTA, 0.02\% $\mathrm{NaN}_{3}, 2$ mM DTT, pH 7.4) and lysed with a French press pressure cell using a minimum pressure of 20,000 psi. The lysate was centrifuged at $38,000 \mathrm{~g}$ for $1 \mathrm{~h}$. The supernatant was filtered and then applied to a column containing $25 \mathrm{ml}$ glutathione Sepharose 4 Fast Flow resin. Protein fractions were eluted with three column volumes of $50 \mathrm{mM}$ Tris- $\mathrm{HCl}, 300 \mathrm{mM} \mathrm{NaCl}, 2.5$ mM EDTA, 0.02\% $\mathrm{NaN}_{3}, 2 \mathrm{mM}$ DTT, pH 7.4, and $10 \mathrm{mM}$ reduced glutathione. Fractions containing the fusion protein were collected, and the GST tag was cleaved using a 1:100 ratio of HRV3C protease. The tag was removed, and the samples were buffer exchanged into $50 \mathrm{mM}$ sodium phosphate, $300 \mathrm{mM} \mathrm{NaCl}, 1 \mathrm{mM}$ EDTA, $0.02 \%$ sodium azide and $2 \mathrm{mM}$ DTT. Samples were then applied to a GE HiLoad 16/60 Superdex75 column as the final purification step.

\section{NMR experiments.}

NMR experiments on free and Mdm2-bound WT and mutant p53TADs were carried out on samples containing $0.1 \mathrm{mM}{ }^{13} \mathrm{C}$ - and ${ }^{15} \mathrm{~N}$-labeled p53TAD (residues 1-73) and $0.5 \mathrm{mM}$ of unlabeled Mdm2 (residues 17-125), at $25^{\circ} \mathrm{C}$ on a Varian VNMRS $600 \mathrm{MHz}$ spectrometer equipped with a triple resonance pulse field Z-axis gradient cold probe. Resonance assignments for WT human p53TAD were previously reported ${ }^{12}$. For amide ${ }^{1} \mathrm{H}$ and ${ }^{15} \mathrm{~N}$, as well as ${ }^{13} \mathrm{C}_{\alpha}$ and ${ }^{13} \mathrm{C}_{\beta}$ resonance assignments of p53TAD mutants, sensitivity-enhanced ${ }^{1} \mathrm{H}_{-}{ }^{15} \mathrm{~N}$ HSQC and threedimensional HNCACB experiments were recorded on uniformly ${ }^{15} \mathrm{~N}$ - and ${ }^{13} \mathrm{C}$-labeled samples in $50 \mathrm{mM}$ sodium phosphate buffer, $100 \mathrm{NaCl}, 1 \mathrm{mM}$ EDTA, $0.02 \%$ sodium azide and $2 \mathrm{mM}$ DTT, at pH $6.8\left(10 \% \mathrm{D}_{2} \mathrm{O}\right)$. For HNCACB experiments, data were acquired along ${ }^{1} \mathrm{H},{ }^{13} \mathrm{C}$ and ${ }^{15} \mathrm{~N}$ dimensions using 7,225.4335 $\left(\mathrm{t}_{3}\right) \times 12,063.4480\left(\mathrm{t}_{2}\right) \times 1,499.9813\left(\mathrm{t}_{1}\right) \mathrm{Hz}$ sweep widths and 
$512\left(\mathrm{t}_{3}\right) \times 128\left(\mathrm{t}_{2}\right) \times 32\left(\mathrm{t}_{1}\right)$ complex data points. The sweep widths and complex points of the HSQC were $7225.4335\left(\mathrm{t}_{2}\right) \times 1500\left(\mathrm{t}_{1}\right) \mathrm{Hz}$ and $512\left(\mathrm{t}_{2}\right) \times 128\left(\mathrm{t}_{1}\right)$, respectively. Resonance assignments for free and p53TAD-bound Mdm2 were obtained with HNCA and HNCACB experiments on samples containing $0.1 \mathrm{mM}{ }^{13} \mathrm{C}$ - and ${ }^{15} \mathrm{~N}$-labeled $\mathrm{Mdm} 2$ (residues 17-125) and $0.5 \mathrm{mM}$ of unlabeled WT p53TAD and p53TAD ${ }^{\mathrm{P} 27 \mathrm{~A}}$, at $25^{\circ} \mathrm{C}$ and $37^{\circ} \mathrm{C}$ on Varian VNMRS 800 and Bruker $750 \mathrm{MHz}$ spectrometers equipped with pulse field Z-axis gradient cold probes, in 50 mM sodium phosphate buffer, $100 \mathrm{NaCl}, 1 \mathrm{mM}$ EDTA, $0.02 \%$ sodium azide and $2 \mathrm{mM}$ DTT, at pH $6.8\left(10 \% \mathrm{D}_{2} \mathrm{O}\right)$. For HNCA and HNCACB experiments, data were acquired in ${ }^{1} \mathrm{H},{ }^{13} \mathrm{C}$ and ${ }^{15} \mathrm{~N}$ dimensions using sweep widths of 13,020.8 $\left(t_{3}\right) \times 6,032.7\left(t_{2}\right) \times 3,000\left(t_{1}\right) \mathrm{Hz}$ and 1,024 $\left(t_{3}\right)$ $\times 128\left(t_{2}\right) \times 32\left(t_{1}\right)$ complex data points. The sweep widths and complex points for 2D HSQC experiments were 9,689.9 $\left(t_{2}\right) \times 2,500\left(t_{1}\right) \mathrm{Hz}$ and 1,024 $\left(t_{2}\right) \times 128\left(t_{1}\right)$. Heteronuclear ${ }^{1} \mathrm{H}^{-15} \mathrm{~N}$ steady-state nuclear Overhauser effect (hNOE) experiments on ${ }^{15} \mathrm{~N}$ isotope-labeled WT and mutant p53TADs $(0.1 \mathrm{mM})$ and unlabeled $\mathrm{Mdm} 2(0.1 \mathrm{mM})$ were recorded in the presence and absence of a $120^{\circ}$ off-resonance ${ }^{1} \mathrm{H}$ saturation pulse every $5 \mathrm{~ms}$ for a total of $3 \mathrm{~s} .512\left(t_{2}\right) \times 128$ $\left(t_{1}\right)$ complex points were recorded with 128 scans per increment. NHNOE values were determined by taking the quotient of the intensity for resolved resonances in the presence and absence of proton saturation. Three measurements were made on each protein, and values were averaged.

All NMR spectra were processed with nmrPipe and analyzed in nmrView ${ }^{26}$. Random coil chemical shift libraries used for secondary chemical shift calculations were generated using the neighbor corrected intrinsically disordered protein database (ncIDP) ${ }^{27}$. As an exception, Gly59, which precedes a proline, required a correction of the random coil chemical shift of -0.77 p.p.m. instead of the -2.0 p.p.m. used in ncIDP $^{28}$.

\section{ITC data collection and analysis.}

ITC experiments were performed on a GE Microcal VP-ITC machine in $50 \mathrm{mM}$ sodium phosphate, $150 \mathrm{mM}$ sodium chloride, $1 \mathrm{mM}$ EDTA, 0.02\% sodium azide, $8 \mathrm{mM} \beta$ mercaptoethanol at pH 6.8. Solutions of WT and mutant p53TAD $(50 \mu \mathrm{M})$ were injected into the sample cell containing recombinant Mmd2 (residues 17-125) at a concentration of $5 \mu \mathrm{M}$ (38 $\times$ $7.5 \mu \mathrm{L}$ ). Sample cells were kept at $25^{\circ} \mathrm{C}$. Data were analyzed with the Origin70 ITC Software. Averages and s.d. from three different ITC experiments are shown. Integrated ITC data were 
fit with single-site binding models, and binding stoichiometries were determined to be between 0.8 and 1.0 .

\section{Time-resolved NMR profiling of WT and mutant p53TAD phosphorylation.}

Phosphorylation of ${ }^{15} \mathrm{~N}$ isotope-labeled WT and mutant p53TAD were monitored by timeresolved NMR spectroscopy as described in ref. 29. All experiments were carried out on a Bruker $750 \mathrm{MHz}$ spectrometer equipped with a cryogenically cooled triple-resonance probe. 2D HMQC-SOFAST NMR spectra were recorded with 70-ms interscan delays, 12,500 $\left(\mathrm{t}_{2}\right) \times 1,976$ $\left(\mathrm{t}_{1}\right) \mathrm{Hz}$ sweep widths and $512\left(\mathrm{t}_{2}\right) \times 32\left(\mathrm{t}_{1}\right)$ complex points. Spectra were processed using linear prediction in the ${ }^{15} \mathrm{~N}$ dimension (32 complex points), cosine-bell apodization and zero filling to 4,096 $\left(\mathrm{t}_{2}\right)$ and 1,024 $\left(\mathrm{t}_{1}\right)$ data points. Phosphorylation levels were determined on the basis of NMR signal intensities in 2D NMR spectra for all phosphorylated residues. Experiments were performed in duplicates. Comparative lysate phosphorylation rates were measured using quenched reaction setups ${ }^{29}$. Extracts were prepared from MCF7 cells containing shRNA-reduced levels of endogenous p53 or stably integrated WT p53 in the shRNA background in the presence of phosphatase inhibitors. Phosphorylation levels of Ser15 and Ser37 were delineated by measuring NMR signal intensities. Modification rates of $\mathrm{p}^{23^{\mathrm{P} 27 \mathrm{~A}}}$ and $\mathrm{p} 53^{\mathrm{P} 3 \mathrm{xA}}$ substrates were determined relative to the normalized phosphorylation rate of WT p53TAD. Experiments were performed in duplicates.

\section{Cell culture and transgenic cell lines.}

MCF7 cells stably expressing p53 shRNA were obtained from the Agami laboratory ${ }^{30}$ and grown in RPMI plus 10\% FBS supplemented with selective antibiotics (400 $\mu \mathrm{g} / \mathrm{ml}$ neomycin and 0.5 $\mu \mathrm{g} / \mathrm{ml}$ puromycin) as appropriate. Using MultiSite Gateway Cloning (Invitrogen), lentiviral vectors (derived from pRRL ${ }^{31}$ ) expressing p53 cDNA fused to the yellow fluorescent protein Venus under the control of the human EF1a promoter were generated. p53 cDNA contained silent mutations to prevent shRNA binding ${ }^{32}$. Mutations of Pro12, Pro13 and Pro27 were introduced by site-directed mutagenesis. VSV-G pseudotyped lentiviral particles were prepared, and after infection, stable clonal cell lines were obtained by selection and limited dilution. To induce DNA damage, cells were treated with 10 Gy $\gamma$-radiation from a cesium 137 source (Heraeus) at a rate of $0.12 \mathrm{~Gy} / \mathrm{s}$ in growth medium without phenol red or riboflavin. 


\section{Antibodies and reagents.}

We used antibodies against p53 (FL393, Santa Cruz, sc-6243, 1:1000), pS15-p53 (Ser15, Santa Cruz, sc-101762, 1:1000), Mdm2 (SMP14, Santa Cruz, sc-965, 1:200) and actin (Sigma, 1:5000). Nutlin 3A and MG132 were obtained from Sigma, TSA was from Invitrogen, and protease and phosphatase inhibitor cocktails were from Carl Roth.

\section{Western blot analysis.}

Protein extracts were prepared by cell lysis in the presence of protease, phosphatase and deacetylase inhibitors. Total protein content was quantified using the BCA assay (Pierce). Equal protein amounts were separated by electrophoreses on 4-12\% Bis-Tris gradient gels (Invitrogen) and transferred onto nitrocellulose membranes by electroblotting. Membranes were blocked with 5\% nonfat dried milk, incubated overnight with primary antibodies and washed and incubated with secondary antibodies coupled to peroxidase. Protein levels were detected using chemoluminiscence (ECL plus, Amersham) after additional washing steps.

\section{Live cell microscopy.}

For live cell time-lapse microscopy, cells were plated in RPMI without phenol red and riboflavin, supplemented with 10\% FBS on poly-D-lysine coated glass-bottom plates (MatTek Corporation). Cells were imaged on a Nikon Ti inverted microscope with a 20× plan apo objective (NA 0.75) using a Hamamatsu Orca R2 camera. The Venus filter set was $500 \mathrm{~nm} / 20$ nm excitation, $515 \mathrm{~nm}$ dichroic beam splitter and $535 \mathrm{~nm} / 30 \mathrm{~nm}$ emission (Chroma). The microscope was enclosed with an environmental chamber controlling temperature, atmosphere $\left(5 \% \mathrm{CO}_{2}\right)$ and humidity. Images were acquired every $15 \mathrm{~min}$ for $12-24 \mathrm{~h}$ using the Nikon Elements software. Image analysis was performed with ImageJ (NIH) and Matlab (MathWorks) as described ${ }^{33}$.

\section{Image analysis and statistics}

Dynamics of p53 levels recorded as time-resolved fluorescence intensity trajectories were analyzed computationally to identify p53 pulses and to characterize statistically significant shape and timing properties. Cells were defined as ‘inducing p53 expression’ when fluorescence levels were higher than the mean of all untreated cells plus 1.5 times the s.d. Frequencies of p53 pulses were analyzed by pitch detection using an autocorrelation function, in which the pitch was 
defined as the time of the first maximum of the autocorrelation function. The pitch score was then determined as the value of the function at this time point. Higher pitch scores indicated more regular pulses, i.e., determinable frequencies. Cells with pitch scores above 0.1 were considered 'oscillating', and their fractions in the various cell lines were counted.

\section{Flow cytometry.}

Cells were trypsinized and fixed in $70 \%$ ethanol at $-20^{\circ} \mathrm{C}$, washed with PBS, incubated with 25 $\mu \mathrm{g} / \mathrm{ml}$ propidium iodide (PI), $0.1 \%$ Triton and $0.2 \mu \mathrm{g} / \mathrm{ml}$ RNase and analyzed on a FACSCalibur or LSRII flow cytometer (BD Biosciences). Cell singlets were identified based on the pulse width/height ratio and used for further analysis. To obtain the percentages of G1, S, G2-M, and endoreduplicated cells, DNA content distributions were computationally fitted using a modification of the Dean-Jett model $^{25}$.

\section{Gene expression analysis.}

RNA was isolated using the HighPure RNA isolation kit (Roche). For RT-qPCR, cDNA was generated using M-MuLV reverse transcriptase (NEB) and oligo-dT primers. Triplicate reactions were set up using the SYBR Green Master Mix (Roche) and run on a StepOnePlus PCR machine (Applied Biosystems). The following primers were used for RT-qPCR: actin forward, GGC ACC CAG CAC AAT GAA GAT CAA; actin reverse, TAG AAG CAT TTG CGG TGG ACG ATG; Bax forward, CTG ACG GCA ACT TCA ACT GG; Bax reverse, GAT CAG TTC CGG CAC CTT GG; p21 forward, TGG ACC TGT CAC TGT CTT GTC; p21 reverse, TCC TGT GGG CGG ATT AG.

For RNA sequencing, RNA quality was analyzed with the Agilent RNA 6000 Nano Kit, and the concentration was measured with the Qubit RNA Assay Kit (Invitrogen). Library preparation was carried out with the TruSeq RNA Sample Preparation Kit (Illumina) using barcoded primers. Libraries were sequenced on an Illumina HiSeq using the single read protocol $(1 \times 100 \mathrm{nt})$. For analyzing the data, we assembled a list of 400 validated p53 target genes from previously published ChIP- and RNA-seq data ${ }^{34,35}$.

26. Johnson, B.A.R. \& Blevins, R.A. NMRView: a computer program for the visualization and analysis of NMR data. J. Biomol. NMR 4, 603-614 (1994).

27. Tamiola, K., Acar, B. \& Mulder, F.A. Sequence-specific random coil chemical 
shifts of intrinsically disordered proteins. J. Am. Chem. Soc. 132, 18000-18003 (2010).

28. Schwarzinger, S. et al. Sequence-dependent correction of random coil NMR chemical shifts. J. Am. Chem. Soc. 123, 2970-2978 (2001).

29. Theillet, F.X. et al. Site-specific NMR mapping and time-resolved monitoring of serine and threonine phosphorylation in reconstituted kinase reactions and mammalian cell extracts. Nat. Protoc. 8, 1416-1432 (2013).

30. Brummelkamp, T.R., Bernards, R. \& Agami, R. A system for stable expression of short interfering RNAs in mammalian cells. Science 296, 550-553 (2002).

31. Dull, T. et al. A third-generation lentivirus vector with a conditional packaging system. J. Virol. 72, 8463-8471 (1998).

32. Gaglia, G., Guan, Y., Shah, J.V. \& Lahav, G. Activation and control of p53 tetramerization in individual living cells. Proc. Natl. Acad. Sci. USA 110, 1549715501 (2013).

33. Loewer, A., Batchelor, E., Gaglia, G. \& Lahav, G. Basal dynamics of p53 reveal transcriptionally attenuated pulses in cycling cells. Cell 142, 89-100 (2010).

34. Menendez, D. et al. Diverse stresses dramatically alter genome-wide p53 binding and transactivation landscape in human cancer cells. Nucleic Acids Res. 41, 7286-7301 (2013).

35. Nikulenkov, F. et al. Insights into p53 transcriptional function via genome-wide chromatin occupancy and gene expression analysis. Cell Death Differ. 19, 1992-2002 (2012). 